\title{
FARADAY ROTATION IN MULTIPLE QUANTUM WELLS OF GaAs/AlGaAs*
}

\author{
E. Dudziak, J. Bożym, D. Pruchnik \\ Institute of Physics, Technical University of Wrocław \\ Wybrzeże Wyspiańskiego 27, 50-370 Wrocław, Poland
}

AND Z.R. WASILEWSKI

Institute for Microstructural Sciences, National Research Council

K1AOR6 Ottawa, Canada

\begin{abstract}
We report on the results of first measurements of the Faraday rotation of modulated $n$-doped multiple quantum wells of $\mathrm{GaAs} / \mathrm{Al}_{x} \mathrm{Ga}_{1-x} \mathrm{As}$ $(x=0.312)$. The measurements have been performed in the magnetic fields up to $13 \mathrm{~T}$ at the temperature of $2 \mathrm{~K}$, in the spectral region of interband transitions. A rich structure of magneto-excitons has been found in the measured spectra. Faraday rotation (phase) measurements are proposed as an alternative method to photoluminescence excitation for investigations of magneto-excitons in quantum wells. The dependence of the measured Faraday rotation on magnetic field and hypothetical connections with quantum Hall effect are also discussed.
\end{abstract}

PACS numbers: 78.20.Ls, 73.20.Dx, 78.66.Fd

\section{Introduction}

Magnetooptics appeared to be very useful in studies of two-dimensional (2D) structures. The method of photoluminescence (PL), photoluminescence excitation (PLE) or modulated reflectance were mostly used [1-7]. Most papers are devoted to single quantum wells. To observe the fine structure in multiple quantum wells (MQW's), discussed in this paper, a very high quality sample is needed. In this report we propose a Faraday rotation (FR) spectroscopy as a method of investigation of the optical excitations in GaAs/AlGaAs MQW's. The measured dependence of the FR angle on magnetic field $\Theta=\Theta(B)$ and the expected connections with quantum Hall effect (QHE) are also discussed.

*The measurements in the magnetic fields have been performed at the International Laboratory of High Magnetic Fields and Low Temperatures, Wrocław, Poland. 


\section{Experiment}

The MQW's were grown on semi-insulating $\langle 100\rangle$ GaAs substrates. The sample under test in this work contained 30 periods of GaAs wells and $\mathrm{Al}_{0.312} \mathrm{Ga}_{0.688} \mathrm{As}$ barriers of thicknesses $183 \AA$ and $402 \AA$, respectively. The barriers were $\delta$-doped with $\mathrm{Si}$ atoms $\left(4 \times 10^{11} \mathrm{~cm}^{-2}\right)$ at the distance $75 \AA$ from one side of the barrier. The measurements have been performed in the reflection configuration, in the visible and near-infrared spectral regions, at temperature of about $2 \mathrm{~K}$. The magnetic field (up to $13 \mathrm{~T}$ ) was perpendicular to the interfaces of MQW's. Light from $100 \mathrm{~W}$ tungsten lamp was passed through a monochromator with a linear polarizer at the exit slit. The polarization state of the light reflected from the sample was analysed by the rotating $(20-40 \mathrm{~Hz}$ ) polarizer placed before the detector (thermocooled Si photodiode with built-in preamplifier). The intensity and the phase of reflected light (relative to the reference phase) were simultaneously measured by two-channel lock-in amplifier.

\section{Results and discussion}

Figure 1a shows some of the FR spectra $\Theta(E)$ at various magnetic fields. At $B=9 \mathrm{~T}$ the $\Theta(E)$ spectrum is compared with the reflectivity curve $R(E)$. We can see the fine structure induced by magnetic field imposed on slowly oscillating background of $R(E)$ (the oscillations result from light interference on thin film). The structure of $R(E)$ corresponds to much more distinct structure of the $\Theta(E)$ spectra, which are the differential spectra $\varepsilon^{-}(E)-\varepsilon^{+}(E)$ of the dielectric function $\varepsilon(E)$ for $\sigma^{ \pm}$circularly polarized light. The presence of interference effects in the measured $R(E)$ curves provides an experimental evidence that the response of our sample is, in fact, transferred by means of the transmitted light which passed doubly through the whole MQW system. In contrast to this, it is worth noting that in the case of PLE the volume of the sample, from which the response signal comes, depends on the penetration depth of the excitation light. Moreover, this excitation beam may modify the measured system.

Our FR spectra (Fig. 1a) like the pseudo-absorption spectra PLE in undoped QW's are dominated by strong Coulomb interaction between electrons and holes. Sharp excitonic peaks correspond to the bound states of electrons and holes of different confined subbands. The fitting of the spectra shape $\Theta(E)$ was performed to identify the energies of transition between different subbands of the valence and conduction bands. The Lorentzian oscillators being the simplest possible dielectric function $\varepsilon(E)$ approximation, appropriate for excitons, has been used. Generally, FR spectra $\Theta(E)$ contain information about transitions for both $\sigma^{+}$and $\sigma^{-}$polarizations. However, mostly when resonance energies $E^{ \pm}$are close to each other, they give one peak (or minimum) with "intrinsic" line width depending on effective $g$-factor. It is a disadvantage of the method.

The transition energies as functions of magnetic field are depicted in Fig. 1b. The areas of the experimental circles are proportional to the oscillator strengths. The transitions in the low energy range originate from the bulk GaAs, probably from buffer region. The exciton line and the first Landau levels are well seen. The higher energy range concerns the transitions in MQW's. The picture of these 

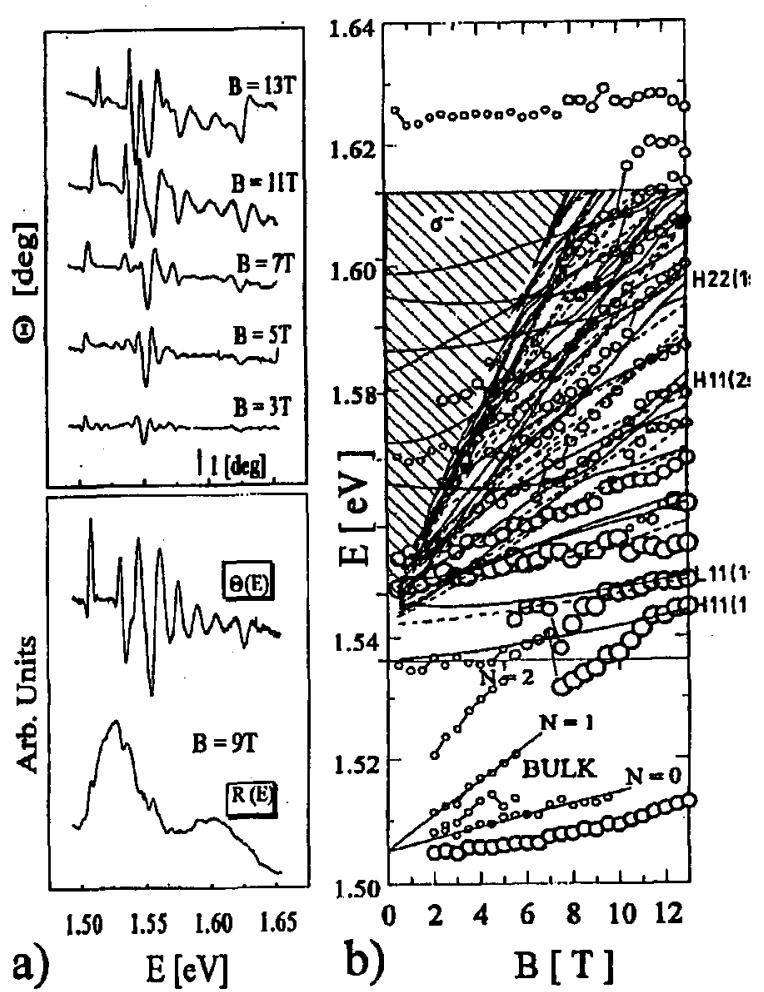

Fig. 1. (a) Exemplary Faraday rotation spectra $\Theta(E)$ at various magnetic fields. The $\Theta(E)$ spectrum is compared with the reflectivity curve $R(E)$ at 9 T. (b) Measured transition energies vs. magnetic field (open circles). The areas of the points are proportional to the oscillator strengths. Solid and dashed lines are the theoretical data from Ref. [5]. The strongest transitions calculated for $\sigma^{-}$polarization are labelled.

transitions differs from the typical Landau level fan chart in a bulk semiconductor. The magneto-exciton structure is dominant. There exists no theory of the magneto-excitons in quantum well which would take into account all factors of the problem: confining potential, the Coulomb interaction, magnetic and electric fields, the Hartree and the exchange-correlation potentials and additionally the electrical plasmons of free carriers. The data from Ref. [5] calculated for $160 \AA$ thick intrinsic quantum well for $\sigma^{-}$polarization have been depicted in Fig. 1b for the sake of qualitative comparison with our experimental results. The simplified theory [8] used in Ref. [5] takes into account only the rectangular confining potential and the exciton states mixing induced by complicated valence-band structure while neglects all second-order many-body effects. The energies of Ref. [5] have been shifted by $+5 \mathrm{meV}$ (roughly estimated effect of Hartree, exchange and correlation energy on intersubband transitions [9]). The chart of our experimental data relatively well reflects the general character of the theoretical picture from Ref. [5]. The 
theoretical solid (dashed) lines correspond to the excitons with $\Gamma_{7}\left(\Gamma_{8}\right)$ symmetry in the convention of Ref. [9]. States belonging to the same irreducible representation can couple. The strongest features correspond to the $n s$ levels of the $\mathrm{H} 1$ and L1 excitons (where $n=1,2 \ldots$ ) except for some cases when the latter interact with the other excitonic states and share their oscillator strengths. The hatched part of Fig. 1b represents a strong mixture of states, which cannot be unambiguously disentangled. The effect of the free carriers plasma on the experimental transitions is particularly evident in the case of the ground states of H11(1s) and L11 $(1 s)$. The transition energies and oscillator strengths abruptly change with a depopulation of the Landau level (Landau level filling factor $\nu=2$ at $B=8.2 \mathrm{~T}$ ). The effect of the excitonic correlations in magnetoplasma is seen in the bending down of the transition lines in the high magnetic field region [10].

From the phenomenological point of view the angle $\Theta$ of $F R$ is proportional to the component of the $\sigma_{x y}$ high frequency conductivity tensor. Therefore in the QHE regime $\left(\sigma_{x x}=\sigma_{y y}=0\right.$ and $\sigma_{x y}=\nu e^{2} / h, \nu$ is the Landau filling factor and the other symbols have the usual meanings) $\Theta$ should (independently of the conductivity mechanism) be equal to $\nu \alpha / n$ where $\alpha=e^{2} / h c$ is the fine structure constant and $n$ is a refractive coefficient. For $\Delta \nu=1$ in QHE regime (e.g. $\nu=2 \rightarrow$ $\nu=1$ ) and for $n=2.5$ (GaAs) the angle of FR should change by $\Delta \Theta=1.12 \mathrm{deg}$ in each quantum well. Extrapolating the experimental dependence $\Theta(B)$ (Fig. 2)

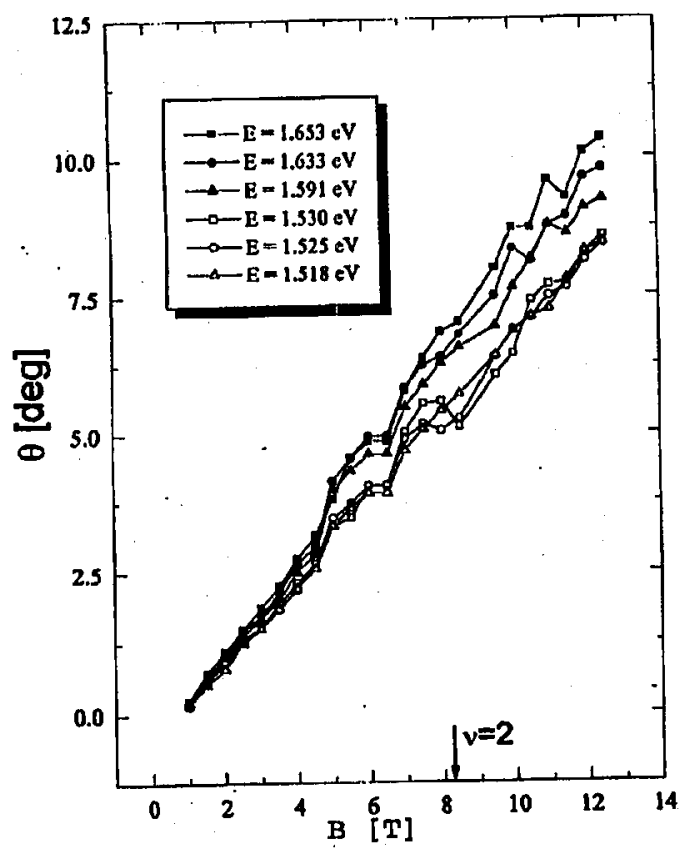

Fig. 2. Faraday rotation vs. magnetic field for different photon energies. The arrow shows calculated $B$ value at Landau filling factor $\nu=2$ for the nominal concentration of $N_{s}=4 \times 10^{11} \mathrm{~cm}^{-2}$. 
to $B \approx 16.4 \mathrm{~T}(\nu=1)$ we get $\Delta \Theta=0.11 \mathrm{deg}$ which agrees quite well with the above estimate.

We observed no reproducible plateaus. Goldberg et al. [11], who observed pinning of the PL emission energy at $\nu=1$ being an optical analog of QHE, did it at temperatures lower than $2 \mathrm{~K}$.

\section{Conclusions}

The FR in MQW's of GaAs/AlGaAs has been measured for the first time. The FR measurements appeared to be a simple and sensitive (differential) method of investigation of magneto-excitons in QW's. This method works in a wide spectral region and does not use a high energy light excitation which may modify the state of the sample. The FR measurements performed in QH regime provide a good and direct opportunity to investigate optical analogs of QHE. Our measurements constitute some contribution to the experimental confirmation of the possible universal character of FR in the regime of QHE.

\section{References}

[1] J.C. Maan, G. Belle, A. Fasolino, M. Altarelli, K. Ploog, Phys. Rev. B 30, 2253 (1984).

[2] L. Vina, G.E.W. Bauer, M. Potemski, J.C. Maan, E.E. Mendez, W.I. Wang, Phys. Rev. B 38, 10154 (1988).

[3] L. Vina, G.E.W. Bauer, M. Potemski, J.C. Maan, E.E. Mendez, W.I. Wang, Phys. Rev. B 41, 10767 (1990).

[4] R. Stępniewski, W. Knap, A. Raymond, G. Martinez, J.C. Maan, B. Estienne, K. Ploog, Surf. Sci. 229, 519 (1990).

[5] L. Vina, G.E.W. Bauer, M. Potemski, J.C. Maan, E.E. Mendez, W.I. Wang, Surf. Sci. 229, 504 (1990).

[6] P.E. Simmonds, M.S. Birket, M.S. Skolnick, W.I.E. Tagg, P. Sobkowicz, G.W. Smith, D.M. Whittaker, Phys. Rev. B 50, 11251 (1994).

[7] T. Yasui, Y. Segava, Y. Aoyagi, Y. Timura, G.E.W. Bauer, I. Mogi, G. Kido, Phys. Rev. $B$ 51, 9813 (1995).

[8] G.E.W. Bauer, T. Ando, Phys. Rev. B 38, 6015 (1988).

[9] W.L. Bloss, J. Appl. Phys. 66, 3639 (1989).

[10] G.E.W. Bauer, Surf. Sci. 229, 374 (1990).

[11] B.B. Goldberg, D. Heinman, A. Pinczuk, L.N. Pfeifer, K.W. West, Phys. Rev. Lett. 65, $641(990)$. 